\title{
A NOTE ON WHITTAKER'S CARDINAL SERIES IN HARMONIC ANALYSIS
}

\author{
by M. M. DODSON, A. M. SILVA and V. SOUCEK
}

(Received 1st April 1985)

The sampling theorem, often referred to as the Shannon or Whittaker-Kotel'nikovShannon sampling theorem, is of considerable importance in many fields, including communication engineering, electronics, control theory and data processing, and has appeared frequently in various forms in engineering literature (a comprehensive account of its numerous extensions and applications is given in [3]). The result states that a band-limited signal, i.e. a real function $f$ of the form

$$
f(t)=\int_{-w}^{w} F(x) e^{-2 \pi i x t} d x
$$

where $w>0$, is under reasonable conditions on the even function $F$, determined by its values on the sampling set $(1 / 2 w) \mathbb{Z}$ and can be reconstructed from the samples $f(k / 2 w)$, $k \in \mathbb{Z}$, by the series

$$
f(t)=\frac{\sin 2 \pi w t}{2 \pi w} \sum_{k \in \mathbb{Z}} f\left(\frac{k}{2 w}\right) \frac{(-1)^{k}}{(t-k / 2 w)}=\frac{1}{2 w} \sum_{k \in \mathbb{Z}} f\left(\frac{k}{2 w}\right) \frac{\sin 2 \pi w(t-k / 2 w)}{\pi(t-k / 2 w)}
$$

This series is known as the cardinal series and was established by E. T. Whittaker [12] in 1915 from the point of view of interpolation theory. In an interesting survey article [2] which also reviews some of the mathematics connected with the cardinal series, Higgins traces the origins of the result to before Whittaker and points out that others besides Kotel'nikov and Shannon appreciated its significance.

The purpose of this note is to combine two different generalisations of the sampling theorem. The first is a far-reaching extension due to Kluvánek ([4]; see also [2], §4) from classical Fourier analysis to abstract harmonic analysis, with the real line $\mathbb{R}$ being replaced by a locally compact abelian group $G$ and its dual $\Gamma$, the interval $(-w, w)$ outside which the Fourier transform vanishes being replaced by a complete set of coset representatives of $\Gamma / \Lambda$ and the discrete subgroup (sampling set) $\mathbb{Z} / 2 w$ by a discrete subgroup $H$ of $G$. In making this extension, Kluvanek notes that in the classical case, when $G=\mathbb{R}$, the set of coset representatives need not be an interval. The second generalisation replaces the interval in the sampling theorem by a somewhat more 
general subset $A$ of $\mathbb{R}$ which is measurable and with difference set $D(A)$ which meets $\mathbb{Z} / s$ just in 0 for some positive number $s$ (the sampling interval)[1]. The equivalent "disjoint translates" condition that each translate $A+k / s$, where $k$ is a non-zero integer, is disjoint from $A$ is used by Lloyd ([6]) in sampling theorems for stochastic processes which are stationary in the wide sense and which have spectral support $A$. Lloyd's results have been extended to a wide class of non-stationary processes and to harmonisable processes indexed by suitable locally compact abelian groups [5]; some of the ideas in [5] are close to those in [4] and the present paper. When placed in an abstract harmonic analysis setting, the difference set and disjoint translates conditions are weaker than Kluvánek's and a specialisation gives an $n$-dimensional sampling result more effective than that in [9]. Note that in (1), $f$ is the Fourier transform of a given function $F$; in the sequel, the function $f$ is taken as given.

We will follow, with some minor modifications, Kluvánek's approach in [4]. In particular instead of starting with a discrete subgroup $H$ of $G$ (corresponding to the subgroup $s \mathbb{Z}$ when $G=\mathbb{R}$ ) and finding a class of functions determined by their values on $H$, we start with a set $A$ outside which the Fourier transforms of the functions vanish and find conditions for a subgroup $H$ to be a sampling set.

Let $G$ be a locally compact abelian group with dual group $\Gamma$. The value of the character (or homomorphism) $\gamma: G \rightarrow S^{1}$ of $\Gamma$ at the point $x$ in $G$ will be written $(x, \gamma)$. For each function $f$ in $L^{1}(G)$, the Fourier integral or transform $f: \Gamma \rightarrow \mathbb{C}$ is defined by

$$
\hat{f}(\gamma)=\int_{G} f(x)(-x, \gamma) d m_{G}(x)
$$

and for each $\psi \in L^{1}(\Gamma)$, the inverse Fourier transform $\bar{\psi}: G \rightarrow \mathbb{C}$ is defined by

$$
\psi(x)=\int_{\Gamma} \psi(\gamma)(x, \gamma) d m_{\Gamma}(\gamma)
$$

The Haar measure $m_{\Gamma}$ on $\Gamma$ is normalised so that the inversion theorem holds (results on integration on locally compact abelian groups used in this paper can be found in [7]).

Let $\Lambda$ be a discrete subgroup of $\Gamma$ such that $\Gamma / \Lambda$ is compact (a connected locally compact abelian group $G$ always contains a discrete finitely generated subgroup $D$ such that the factor group $G / D$ is compact $([8], \S 2.21))$. The Haar measure $m_{\Lambda}$ on $\Lambda$ as usual satisfies $m_{\Lambda}(\{\lambda\})=1$ for each $\lambda$ in $\Lambda$. Since $\Gamma / \Lambda$ is compact, the Haar measure $m_{\Gamma / \Lambda}(\Gamma / \Lambda)$ is finite but will not be taken to be 1 and instead by [7], $\S 33 \mathrm{~A}$ or [11], $\$ 2.7 .3$ is normalised so that

$$
\int_{\Gamma} F(\gamma) d m_{\Gamma}(\gamma)=\int_{\Gamma / \Lambda} \sum_{\lambda \in \Lambda} F(\gamma+\lambda) d m_{\Gamma / \Lambda}([\gamma])
$$

holds for every non-negative Baire and so every integrable function $F$ on $\Gamma([\gamma]=\gamma+\Lambda$, the coset of $\Lambda$ in $\Gamma$ containing $\gamma$ ). This amounts to assigning $\Gamma / \Lambda$ the measure of a complete set of coset representatives in $\Gamma$.

Let $H$ be the dual of $\Gamma / \Lambda$, so that $H$ is a discrete subgroup of $G$ with point measure 1 and with annihilator $\{\gamma \in \Gamma:(h, \gamma)=1$ for all $h \in H\}=\Lambda$ ([11], Theorem 2.1.2). Every 
character of $\Gamma / \Lambda$ is of the form $(h,[\gamma])$ for some $h$ in $H, \gamma$ in $\Gamma([7], \S 35 \mathrm{~B})$ and the set of characters forms a complete orthogonal family of functions in $L^{2}(\Gamma / \Lambda)([7], \S 38 \mathrm{C})$.

It is readily verified that a set $\Omega$ in $\Gamma$ is a complete set of coset representatives of $\Gamma / \Lambda$ if and only if for each $\gamma$ in $\Gamma, \Omega$ meets $\Lambda+\gamma$ in just one point, i.e. for each $\gamma$ in $\Gamma$,

$$
\operatorname{Card}\{\Omega \cap(\Lambda+\lambda)\}=1 \text {. }
$$

Hence for any complete set $\Omega$ of coset representatives of $\Gamma / \Lambda$,

$$
\sum_{\lambda \in \Lambda} x_{\Omega}(\lambda+\gamma)=1
$$

for each $\gamma$ in $\Gamma$. Let $\Omega$ be a complete set of coset representatives for $\Gamma / \Lambda$ and suppose that $\Omega$ is measurable and $m_{\Gamma}(\Omega)=\mu$ say. It follows that $x_{\Omega}$ is in $L^{1}(\Gamma) \cap L^{2}(\Gamma)$, so that by Plancherel's theorem, $\check{x}_{\Omega} \in L^{2}(G)$, is continuous, positive definite with norm $\left\|\check{x}_{\Omega}\right\|_{G}$ satisfying

$$
\left\|\check{x}_{\Omega}\right\|_{G}^{2}=\left\|x_{\Omega}\right\|_{\Gamma}^{2}=\int_{\Gamma}\left|\dot{x_{\Omega}}(\gamma)\right|^{2} d m_{\Gamma}(\gamma)=\int_{\Gamma} x_{\Omega}(\gamma) d m_{\Gamma}(\gamma)=m_{\Gamma}(\Omega)=\mu
$$

Evidently

$$
\check{x}_{\Omega}(0)=\int_{\Gamma} x_{\Omega}(\gamma) d m_{\Gamma}(\gamma)=m_{\Gamma}(\Omega)=\mu
$$

Equations (2) and (4) imply that the set of characters $(h, \gamma)$, where $h \in H$ and $\gamma$ is restricted to $\Omega$, form a complete orthogonal family for $L^{2}(\Omega)$. Writing $F_{1}([\gamma])=F(\gamma)$ for a function $F$ defined on $\Omega$ and using (2) and (4), we have

$$
\begin{aligned}
\int_{\mathbf{\Omega}} F(\gamma) d m_{\Gamma}(\gamma) & =\int_{\Gamma} x_{\mathbf{\Omega}}(\gamma) F(\gamma) d m_{\Gamma}(\gamma)=\int_{\Gamma / \Lambda} \sum_{\lambda \in \Lambda} x_{\Omega}(\gamma+\lambda) F(\gamma+\lambda) d m_{\Gamma / \Lambda}([\gamma]) \\
& =\int_{\Gamma / \Lambda} F_{1}([\gamma]) d m_{\Gamma / \Lambda}([\gamma]) .
\end{aligned}
$$

It is clear that $F$ is in $L^{1}(\Omega)$ if and only if $F_{1}$ is in $L^{1}(\Gamma / \Lambda)$; similarly $F$ is in $L^{2}(\Omega)$ if and only if $F_{1}$ is in $L^{2}(\Gamma / \Lambda)$. Since $(h, \gamma)_{1}=(h,[\gamma])_{1}$, the assertion is proved. It follows that for each $h$ in $H$,

$$
\check{x}_{\mathbf{\Omega}}(h)=\int_{\Gamma} x_{\mathbf{\Omega}}(\gamma)(h, \gamma) d m_{\Gamma}(\gamma)=\int_{\Omega}(h, \gamma) d m_{\Gamma}(\gamma)= \begin{cases}0 & \text { when } h \neq 0 \\ \mu & \text { when } h=0 .\end{cases}
$$

Also, by Parseval's formula,

$$
\left.\int_{G} \check{x}_{\Omega}(x) \check{x}_{\Omega}(h-x) d m_{G}(x)=\int_{\Gamma} x_{\Omega}(\gamma) \overline{(-h, \gamma}\right) x_{\Omega}(\gamma) d m_{\Gamma}(\gamma)=\int_{\Gamma} x_{\Omega}(\gamma)(\overline{-h, \gamma}) d m_{\Gamma}(\gamma)
$$

so that for each $h$ in $H$,

$$
\int_{G} \check{x}_{\Omega}(x) \check{x}_{\Omega}(h-x) d m_{G}(x)=\check{x}_{\Omega}(h) .
$$



(2),

The Haar measure of $\Gamma / \Lambda$ satisfies $m_{\Gamma / \Lambda}(\Gamma / \Lambda)=m_{\Gamma}(\Omega)=\mu$, since on putting $F=x_{\Omega}$ in

$$
\begin{aligned}
m_{\Gamma}(\Omega) & =\int_{\Gamma} x_{\Omega}(\gamma) d m_{\Gamma}(\gamma)=\int_{\Gamma / \Lambda} \sum_{\lambda \in \Lambda} x_{\Omega}(\lambda+\gamma) d m_{\Gamma / \Lambda}([\gamma]) \\
& =\int_{\Gamma / \Lambda} 1 . d m_{\Gamma / \Lambda}([\gamma])=m_{\Gamma / \Lambda}(\Gamma / \Lambda)=\mu .
\end{aligned}
$$

Let $A$ be a subset of $\Gamma$ and $\Lambda$ a subgroup of $\Gamma$. It is straightforward to verify that for any subset $A$ of $\Gamma$ the following conditions are equivalent:

(i) $A$ is a subset of a complete set of coset representatives for $\Gamma / \Lambda$,

(ii) for each $\gamma$ in $\Gamma$, Card $\{A \cap(\Lambda+\gamma)\} \leqq 1$,

(iii) for each non-zero $\lambda$ in $\Lambda, A \cap(A+\lambda)$ is empty,

(iv) $D(A) \cap \Lambda=\{0\}$.

The group $\Lambda$ corresponds to $(1 / s) \mathbb{Z}$ in [1] and [6]; (ii) is a weaker condition than (3); (iii) corresponds to the disjoint translates condition preventing "aliasing" in [6], while (iv) corresponds to the difference set condition in [1] which ensures that the map $\Phi: L^{2}(\mathbb{R}) \rightarrow l^{2}(\mathbb{R})$ given by $f \rightarrow\left\{s^{-1 / 2} \hat{f}(s k): k \in \mathbb{Z}\right\}$ is an isometry when restricted to $A$ and so has a left inverse.

In the proof of the extension of Kluvanek's result [4], we consider square integrable functions on $G$ with Fourier transforms which vanish outside a measurable subset $A$ of $\Gamma$, i.e., functions in

$$
L_{A}^{2}=\left\{f \in L^{2}(G): \hat{f}(\gamma)=0 \text { almost everywhere when } \gamma \notin A\right\},
$$

the $A$-spectral set of $L^{2}(G)$, when $A$ is a subset of a complete set of coset representatives of $\Gamma$. The main differences from [4] are the different normalisations and the construction of the set $\tilde{A}$ of coset representatives of $\Gamma / \Lambda$ containing $A$.

Theorem 1. Let $G$ be a locally compact abelian group with dual group $\Gamma$. Let $A$ be $a$ measurable subset of $\Gamma$ and suppose that there exists a discrete countable subgroup $\Lambda$ of $\Gamma$ such that for each non-zero $\lambda$ in $\Lambda, A \cap(A+\lambda)$ is empty. Suppose further that $\Gamma / \Lambda$ is compact and has a complete set of coset representatives which is measurable. Then there exists a complete set $\tilde{A}$ of coset representatives which is measurable and contains $A$; and for each $f$ in $L^{2}(G)$ with Fourier transform $\hat{f}$ vanishing almost everywhere outside $A$,

$$
\|f\|_{G}^{2}=\int_{G}|f(x)|^{2} d m_{G}(x)=\mu^{-1} \sum_{h \in H}|f(h)|^{2}
$$

where $H$ is the dual of $\Gamma / \Lambda$ (or the annihilator of $\Lambda$ ) and $\mu=m_{\Gamma}(\tilde{A})$, and $f$ is equal almost everywhere on $G$ to a continuous function. When $f$ is continuous

$$
f(x)=\mu^{-1} \sum_{h \in H} f(h) \check{x}_{A}(x-h)
$$

absolutely and uniformly in $G$. 
Proof. Let $\Omega$ be a complete measurable set of coset representatives of $\Gamma / \Lambda$. The translates $\Omega+\gamma, \gamma \in \Gamma$, cover $\Gamma$ and are measurable, their intersections $A \cap(\Omega+\gamma)$ with $A$ and their translates $A_{\gamma}=A \cap(\Omega+\gamma)-\gamma$ are also measurable. Since for each non-zero $\lambda$ in $\Lambda, A \cap(A+\lambda)$ is empty, the set

$$
\tilde{A}=A \cup\left\{\Omega \backslash \bigcup_{\lambda \in \Lambda} A_{\lambda}\right\}
$$

is a complete set of coset representatives of $\Gamma / \Lambda$ containing $A$, and since $\Lambda$ is countable, $\tilde{A}$ is measurable.

By hypothesis and Plancherel's theorem, $\hat{f} \in L^{2}(\Gamma)$. Since $\hat{f}(\gamma)=0$ for almost all $\gamma$ not in $A \subseteq \widetilde{A}$, there exist complex numbers $a_{h}, h \in H$, such that

$$
\hat{f}(\gamma)=\sum_{h \in H} a_{h}(h, \gamma) x_{A}(\gamma)=\sum_{h \in H} a_{h}(h, \gamma) x_{\tilde{A}}(\gamma),
$$

where the convergence is in the $L^{2}(\tilde{A})$ norm. Hence on taking the inverse Fourier transform,

$$
\check{f}(x)(=f(x))=\sum_{h \in H} a_{h} \int_{\Gamma}(h, \gamma) x_{A}(\gamma)(x, \gamma) d m_{\Gamma}(\gamma)=\sum_{h \in H} a_{h} \int_{\Gamma}(h, \gamma) x_{\bar{A}}(\gamma)(x, \gamma) d m_{\Gamma}(\gamma)
$$

and so

$$
f(x)=\sum_{h \in H} a_{h} \check{x}_{A}(h+x)=\sum_{h \in H} a_{h} \check{x}_{A}(h+x)
$$

in $L^{2}(G)$. Hence

$$
\begin{aligned}
\|f\|_{G}^{2} & =\int_{G}|f(x)|^{2} d m_{G}(x)=\int_{G} \sum_{h \in H} \sum_{h^{\prime} \in H} a_{h} \bar{a}_{h^{\prime}} \check{x}_{\bar{A}}(x+h) \overline{\tilde{x}_{\bar{A}}\left(x+h^{\prime}\right)} d m_{G}(x) \\
& =\sum_{h \in H} \sum_{h^{\prime} \in H} a_{h} \bar{a}_{h^{\prime}} \int_{G} \check{x}_{\bar{A}}(x+h) \overline{\tilde{x}_{\tilde{A}}\left(x+h^{\prime}\right)} d m_{G}(x) \\
& =\sum_{h \in H} a_{h} \bar{a}_{h^{\prime}} \int_{G}\left|\check{x}_{\bar{A}}(x)\right|^{2} d m_{G}(x)=\mu \sum_{h \in H}\left|a_{h}\right|^{2}
\end{aligned}
$$

by (5), the translation invariance of Haar measure and (4).

Since $m_{\Gamma}(\tilde{A})$ is finite, $L^{2}(\tilde{A}) \subseteq L^{1}(\tilde{A})$, so that for each $f$ in $L^{2}(G), \hat{f} \in L^{1}(\tilde{A})$ and $\hat{f}$ is a continuous function of $x$. Thus by the inversion formula, $f$ is equal almost everywhere to a continuous function, so that when $f$ is continuous

$$
f(x)=\sum_{h \in H} a_{h} \check{x}_{A}(x+h)
$$

pointwise by (9). The convergence of the series is absolute from (10), which holds for $\check{x}_{A}$, and Cauchy's inequality. The convergence of the series to $f$ is uniform since for an 
arbitrary finite subset $H_{1}$ of $H$,

$$
\begin{aligned}
\left|f(x)-\sum_{h \in H_{1}} a_{h} \tilde{x}_{A}(x+h)\right| & =\left|\int_{\Gamma} f(\gamma)(x, \gamma) d m_{\Gamma}(\gamma)-\int_{\Gamma} \sum_{h \in H_{1}} a_{h} x_{A}(\gamma)(x+h, \gamma) d m_{\Gamma}(\gamma)\right| \\
& =\left|\int_{\Gamma} \sum_{h \in H} a_{h}(h, \gamma) x_{A}(\gamma)(x, \gamma) d m_{\Gamma}(\gamma)-\int_{\Gamma} \sum_{h \in H_{1}} a_{h} x_{A}(\gamma)(x, \gamma)(h, \gamma) d m_{\Gamma}(\gamma)\right| \\
& =\left|\int_{\Gamma}(x, \gamma) \sum_{h \in H \backslash H_{1}} a_{h}(h, \gamma) x_{A}(\gamma) d m_{\Gamma}(\gamma)\right| \\
& \leqq \int_{\Gamma}\left|\sum_{h \in H \backslash H_{1}} a_{h}(h, \gamma) x_{A}(\gamma)\right| d m_{\Gamma}(\gamma),
\end{aligned}
$$

which, because convergence in $L^{2}(\tilde{A})$ implies convergence in $L^{1}(\tilde{A})$, can by (8) be made arbitrarily small for a suitable choice of $H_{1}$.

Finally to determine $a_{h}$, note that by (9)

$$
f\left(h^{\prime}\right)=\sum_{h \in H} a_{h} \check{x}_{A}\left(h+h^{\prime}\right)=a_{-h^{\prime}} m_{\mathrm{r}}(\tilde{A})=a_{-h^{\prime}} \mu,
$$

by (5) and (6) with $\Omega=\tilde{A}$, whence the result.

It is possible to prove absolute convergence and the formula for $\|f\|_{G}$ without the inversion formula, which requires a complete set of coset representatives. However it appears that the inversion formula is needed to establish uniform convergence.

The sampling theorem has been extended to $n$ dimensions for continuous functions with bounded spectra, i.e. with Fourier transforms vanishing outside a bounded region in $\mathbb{R}^{n}([2], \S 4-5,[9],[10])$. By considering the special case $G=\Gamma=\mathbb{R}^{n}$ with Lebesgue measure and with character $(x, \gamma)$ given by

$$
(x, \gamma)=e^{2 \pi i x \cdot \gamma}
$$

where $x, \gamma \in \mathbb{R}^{n}$ and $x \cdot \gamma=x_{1} \gamma_{1}+\cdots+x_{n} \gamma_{n}$, the result just proved has as a corollary a generalisation of the $n$-dimensional sampling theorem to continuous functions with Fourier transforms vanishing outside a set which satisfies a disjoint translates or equivalent condition, and to a generalisation of Theorem 5 in [1] to $n$-dimensions.

Corollary. Suppose that the Fourier transform $\hat{f}: \mathbb{R}^{n} \rightarrow \mathbb{C}$ of the continuous function $f$ in $L^{2}\left(\mathbb{R}^{n}\right)$ vanishes outside a measurable set $A \subset \mathbb{R}^{n}$. Suppose also that, for some positive real numbers $s_{1}, \ldots, s_{n}$,

$$
A \cap\left\{A+\left(k_{1} / s_{1}, \ldots, k_{n} / s_{n}\right)\right\}=\phi
$$


holds for all non-zero integer vectors $\left(k_{1}, \ldots, k_{n}\right)$ in $\mathbb{Z}^{n}$. Then

$$
f(x)=\left(s_{1} \ldots s_{n}\right) \sum_{k \in \mathbb{Z}^{*}} f\left(s_{1} k_{1}, \ldots, s_{n} k_{n}\right) \check{x}_{A}\left(x_{1}-s_{1} k_{1}, \ldots, x_{n}-s_{n} k_{n}\right)
$$

uniformly and absolutely.

Proof. The locally compact abelian group $\mathbb{R}^{n}$ with Lebesgue measure is its own dual. The group

$$
\Lambda=\mathbb{Z} / s_{1} \oplus \cdots \oplus \mathbb{Z} / s_{n}
$$

is a discrete and countable subgroup of $\mathbb{R}^{n}$ and the factor group

$$
\mathbb{R}^{n} / \Lambda=\frac{1}{s_{1}} \mathbb{R} / \mathbb{Z} \oplus \cdots \oplus \frac{1}{s_{n}} \mathbb{R} / \mathbb{Z},
$$

is compact and has a complete set of coset representatives given by

$$
\Omega=[0,1) / s_{1} \times \cdots \times[0,1) / s_{n}
$$

with Lebesgue measure $|\Omega|=\left(s_{1} \ldots s_{n}\right)^{-1}$. The map $v: \mathbb{R}^{n} \rightarrow \Omega$ given by

$$
v\left(x_{1}, \ldots, x_{n}\right)=\left(x_{1}-\left[s_{1} x_{1}\right] / s_{1}, \ldots, x_{n}-\left[s_{n} x_{n}\right] / s_{n}\right)
$$

where $[t]$ is the integer part of the real number $t$, is differentiable almost everywhere with Jacobian 1 and is surjective. It is readily verified that the set $A \cup\{x \in \Omega: v(x) \notin v(A)\}$ is a complete set of coset representatives of $\mathbb{R}^{n} / \Lambda$ containing $A$ and corresponds to the set $\tilde{A}$ in the theorem. When restricted to $\tilde{A}=A \cup\{x \in \Omega: v(x) \notin v(A)\}$, the map $v$ is injective and preserves measure, so that $|\tilde{A}|=|v(\tilde{A})|=|\Omega|=\left(s_{1} \ldots s_{n}\right)^{-1}$. The corollary follows since the dual of $\mathbb{R}^{n} / \Lambda$ is the discrete group

$$
H=s_{1} \mathbb{Z} \oplus \cdots \oplus s_{n} \mathbb{Z}
$$

As an example let $n=2$ and let

$$
A=\{(x, y): \max [|x \pm 10|,|y|\}<1 / 2\} \cup\{(x, y): \max \{|x|,|y \pm 10|\}<1 / 2\}
$$

so that $A$ consists of four squares each with unit width lying on a coordinate axis and centred a distance 10 units from the origin. It can be verified that for any pair of integers $j, k$, not both $0, A+3(j, k)$ is disjoint from $A$, so that we can take $s_{1}=s_{2}=1 / 3$, corresponding to a sampling density of 9 . Hence if $f$ is continuous and $\hat{f}$ vanishes almost everywhere outside $A$, then

$$
f(x, y)=\frac{1}{9} \sum_{j, k^{*} \in \mathbb{Z}} f\left(\frac{1}{3}(j, k)\right) \check{x}_{A}\left(x-\frac{1}{3} j, y-\frac{1}{3} k\right)
$$


where

$$
\begin{aligned}
\check{x}_{A}(x, y) & =\int_{A} e^{2 \pi i(x u+y v)} d u d v \\
& =\frac{\sin \pi x(\sin 21 \pi y+\sin 19 \pi y)+\sin \pi \mathrm{y}(\sin 21 \pi x+\sin 19 \pi x)}{\pi^{2} x y} .
\end{aligned}
$$

Note that $|A|=4<9=|\tilde{A}|$.

- By comparison, the $n$-dimensional sampling theorem [10] gives $s_{1}=s_{2}=1 / 21$, corresponding to a sampling density of 441 , but has the simpler interpolation function

$$
\frac{4 \sin 21 \pi x \sin 21 \pi y}{(21)^{2} \pi^{2} x y} .
$$

In this theorem, the spectrum is enclosed in an $n$-dimensional cube centred at the origin; in the above example, the square has sides of length 21. Petersen and Middleton ([9], see also [2], §5) have improved on the $n$-dimensional sampling theorem by enclosing the spectrum in a "cell" which is then "packed" in $\mathbb{R}^{n}$. They do not discuss convergence but their arguments make no essential use of the enclosing cells which could be replaced by a disjoint translates condition. Nevertheless there are some practical advantages in working with cells and it evidently guarantees that translates of the spectrum by vectors corresponding to the packing lattice do not overlap. By considering a diamond shaped cell or by rotating the axes through $\pi / 4$ and then using the $n$-dimensional sampling theorem, it can be shown that the function is given by its values at the lattice points $2 \sqrt{ } 2 /(21+\sqrt{ } 2)(j+k, j-k)$, where $j, k$ are integers, corresponding to $s_{1}=s_{2}=\sqrt{ } 2 /(21+\sqrt{ } 2)=$ $0.063 \ldots$ and sampling density of $251.198 \ldots$, with interpolation function

$$
\frac{8 \sin (21+\sqrt{ } 2) \pi(x+y) / \sqrt{ } 2 \sin (21+\sqrt{ } 2) \pi(x-y) / \sqrt{ } 2}{(21+\sqrt{ } 2)^{2} \pi^{2}\left(x^{2}-y^{2}\right)} .
$$

A more efficient packing is obtained by taking the cell to be the cross

$$
\{(x, y):|x| \leqq 1 / 2,|y| \leqq 21 / 2\} \cup\{(x, y):|x| \leqq 21 / 2,|y| \leqq 1 / 2\}
$$

with arms of length $21 / 2$ and width 1 . It can be verified that distinct translates of the cross by $j(11,1)+k(1,12)=(11 j+k, j+12 k)$, where $j, k$ are integers, do not overlap. In this case Petersen and Middleton's method gives a sampling density of 131 and a corresponding interpolation function

$$
\frac{\sin \pi x \sin 21 \pi y+\sin 21 \pi x \sin \pi y-\sin \pi x \sin \pi y}{\pi^{2} x y}
$$

The group $\mathbb{Z} / s_{1} \oplus \cdots \oplus \mathbb{Z} / s_{n}$ in the corollary was chosen for simplicity and in a particular application, this group could be replaced by one more suited to the structure of the set $A$. Thus as an example when the set $A$ is the cross (12), the appropriate group 
is $\Lambda=\{(11 j+k, j+12 k): j, k \in \mathbb{Z}\}$ with annihilator (sampling set) $H=\{(12 j-k, 11 k-j)$ / $131: j, k \in \mathbb{Z}\}$ and the corresponding interpolation function is given by (13).

Acknowledgements. We are grateful to the referee for some useful comments. M.M.D. is very grateful to the British Council for supporting a visit to Charles University and to the Czechoslovakian Ministry of Education for its help and hospitality.

\section{REFERENCES}

1. M. M. Dodson and A. M. Silva, Fourier analysis and the sampling theorem, Proc. Roy. Irish Acad. 85A (1985), 81-108.

2. J. R. Higgins, Five short stories about the cardinal series, Bull. Amer. Math. Soc. 12 (1985), 45-89.

3. A. J. JeRrI, The Shannon sampling theorem-its various extensions and applications: a tutorial review, Proc. IEEE 65 (1977), 1565-1596.

4. I. KLUVÁNEK, Sampling theorem in abstract harmonic analysis, Matematicko-Fyzikálny Casopis 15 (1965), 43-48.

5. A. J. LEE, Sampling theorems for nonstationary stochastic processes, Trans. Amer. Math. Soc. 242 (1978), 225-241.

6. S. P. Lloyd, A sampling theorem for stationary (wide sense) stochastic processes, Trans. Amer. Math. Soc. 92 (1959), 1-12.

7. L. H. Loomis, An Introduction to Abstract Harmonic Analysis (Van Nostrand, New York, 1953).

8. D. MONTGOMERY and L. ZIPPIN, Topological Transformation Groups (Interscience, New York, 1955).

9. D. P. Petersen, and D. Middleton, Sampling and reconstruction of wave-number-limited functions in $N$-dimensional Euclidean spaces, Inform. and Control 5 (1962), 279-323.

10. F. M. ReZA, An Introduction to Information Theory (McGraw-Hill, New York, 1961).

11. W. Rudin, Fourier Analysis on Groups (Interscience, New York, 1962).

12. E. T. Whittaker, On the functions which are represented by the expansions of the interpolation theory, Proc. Roy. Soc. Edinburgh 35 (1915), 181-194.

\author{
Mathematics Department \\ UNIVERSITY OF YORK \\ HesLington \\ YORK Y01 5DD \\ ENGLAND
}

Katedra Matematicke Analyzy

Charles University

SoKolOVSKa 83

18600 P RAHA 8

CZEChOSLOVAKIA
Departmento de Fisica Universitad Nacional de la Plata CaLle 115 y $49-$ CC No 67 1900 La Plata ARgentina 